\title{
Communication mediums used by clients and health professionals in accessing and providing healthcare in low resource settings: A descriptive cross-sectional study
}

Mathias Dongyele ( $\square$ matino242410@gmail.com )

University of Science and Technology College of Medicine and Health Sciences https://orcid.org/00000002-7547-7159

\section{Daniel Ansong}

Kwame Nkrumah University of Science and Technology

Francis Adjei Osei

Komfo Anokye Teaching Hospital

\section{Sam Newton}

Kwame Nkrumah University of science and Technology, School of Public Health

\section{Evans Xorse Amusu New}

Komfo Anokye Teaching Hospital

Nicholas Karikari Mensah New

Komfo Anokye Teaching Hospital

Alfred Kwame Owusu New

Komfo Anokye Teaching Hospital

\section{Bright Atta Dunkwa New}

Komfo Anokye Teaching Hospital

\section{Samuel Odoom Frimpong}

Research and Development Unit, KATH

Joseph Bonney

Komfo Anokye Teaching Hospital

\section{Duncan Adjei}

Research Development Unit, KATH, Kumasi, Ghana

\section{Anne Amusu}

University of Ghana School of Medicine and Dentistry

\section{Emmanuel Kofi Akowuah}

Department of Computer Engineering, Kwame Nkrumah University of Science and Technology

Kelvin Lartey

Department of computer Engineering, Kwame Nkrumah University of Science and Technology, kumasi, Ghana 


\section{Christopher Seth Yaw Appiah}

Ludwig-Maximilians-Universitat Munchen

\section{Research article}

Keywords: Communication, hospital, clients, healthcare, Ghana

Posted Date: April 1st, 2020

DOI: https://doi.org/10.21203/rs.2.14329/v4

License: (c) (1) This work is licensed under a Creative Commons Attribution 4.0 International License. Read Full License

Version of Record: A version of this preprint was published at Advances in Public Health on May 8th, 2021. See the published version at https://doi.org/10.1155/2021/7419305. 


\section{Abstract}

Background: There exist continuing challenges with communication media used during health service provision. These challenges relate to clients and health institution, intra and inter-health institution communications. This study reviewed the existing healthcare communication media from the perspectives of clients and health professionals at a tertiary hospital in Ghana. Method: cross-sectional design was employed with a multilevel sampling method to select a total of 651 participants consisting of 304 clients, 303 health workers and 44 hospital directorate managers for the study. A well-structured survey questionnaire was used to collect data from respondents. Results: Two thirds of the hospital staff $(66.4 \%)$ used a blend of social media and direct means (face-to-face medium) to communicate among themselves. The majority (89.8\%) of the staff communicated with management through meetings sections. Nearly all healthcare providers $(97.4 \%)$ communicated with clients through direct means (faceto-face medium). Almost all forms of communication between the hospital management members and the general public was done through letters and official memos. Conclusions: The study revealed blended forms of communication media used by health providers and health service consumers. Delayed information flow and poor feedbacks contributed to the dissatisfaction levels associated with the traditional communication media platforms used.

\section{Background}

Client-centered healthcare delivery that demonstrates effectiveness remains a major contributor to the achievement of the 'one health for all' concept (1). In this regard, clients and their relatives along with health providers remain pivotal to the overall health decision making process. The available clinical services and means of healthcare require the adoption of the communication medium by both providers and clients for easy understanding and interactions.

Globally, health transforming policies and strategic plans have been laid down by several countries to help position them to build an informative and knowledge-based economy. Ghana is making significant progress in this direction (2). The advantage of the information and knowledge-based economy driven on effective communication system is that, it serves as a platform for further integrated economy. Communicating using the mobile phone as a medium for information sharing across the healthcare divide has proven to be very effective $(3,4)$. There have been improvements in the use of mobile phone in healthcare delivery among health experts. This medium has seen a quick advancement in the development of effective communication systems. This has therefore facilitated better communication and information sharing at the hospital wards $(3,4)$.

In Ghana, mediums for communication and information sharing have been known to play a critical role during communication among professionals and personnel in organizations most especially in health service delivery points(5). Due to this positivity, interventions such as $\mathrm{m}$-Health have been tested and proven to be effective in the Ghanaian health system, even though the system is yet to be scaled up and adopted in its entirety by the nation(5). Despite the importance and recommendations by major health 
players like the World Health Organization to explore the adoption of communication mediums in Ghana to improve healthcare system delivery, there continue to remain bottlenecks in its adoption and implementation.

The associated challenges in the health system include problems with identifying the nearest hospital, type of services available, availability of physicians and beds at the receiving hospitals as well as lack of reminder systems for providers and clients accessing tertiary facilities like the Komfo Anokye Teaching Hospital (KATH) (6-8). KATH is one of the major healthcare delivery centres in Ghana though the hospital currently does not have a functional hospital-client driven communication medium which allows for effective clients-staff interactions for efficient service delivery (9). Evidence-based information on the communication mediums used by staffs and users of the facility are required for planning and policy making for enhanced efficiency.

While the need for evidence to inform health communication management policy remains pivotal, there is little known about the type of communication that would best serve the needs of hospital management. It remains uncertain whether the anecdotal evidence on the existing communication mediums used in the hospital are effective from multi-stakeholder perspective. Effectiveness would measure the extent to which the communication media used are able to achieve the goals for which they were primarily adopted within the context of healthcare management, internal communication and client-physician engagement. It is on the basis of identifying what mediums are used, how effective they have been and what is considered the preferred medium to be used from the varying perspectives of healthcare workers, clients and hospital management that this study is timely. This study sought to identify the communication media used by clients-staff, staff-staff and staff-management at the Komfo Anokye Teaching Hospital when seeking and providing health services respectively.

\section{Methods}

\section{Study design}

The study employed a cross-sectional design. A multilevel sampling method was used to select a total of 651 participants consisting of 30 clients, 303 health workers and 44 hospital directorate managers for the study. A well-structured survey questionnaire was used to collect data from respondents.

\section{Setting}

The study was conducted at the Komfo Anokye Teaching Hospital (KATH), Kumasi, Ghana from October to November 2018. The hospital is located in the vibrant and culturally rich city of Kumasi, the regional capital of Ashanti, with a population of about 4.7 million(10). It is the second largest hospital in Ghana, and the only tertiary institution in the Ashanti Region. It serves as a major referral centre for the northern sector of Ghana. The hospital has a staff population of 3,909 who fall under these categories; Doctors (9.4\%), Top Management (0.2\%), Nurses and Midwives (42.2\%), Certified Registered Anaesthetist (1.3\%), 
Pharmacist and Pharmacy technicians (3.8\%), Administration and Finance (6.6\%), Clinical support staff (10.9\%) and Allied Health personnel (5.6\%)

\section{Participants}

The study participants enrolled included health workers (doctors, nurses, pharmacists, Allied health staff, clinical support staff and directorates managers) who had worked at KATH for at least five years. The rationale for the inclusion of these study participants was because they had either had and or continue to have direct communication with the clients during consultation, diagnostics and treatment. Staff without direct engagement with health seeking clients and were neither part of the administrative management team of the hospitals were excluded. The out-patients seeking healthcare and treatments at the hospital were also included in the study. Terminally ill clients were excluded as they were deemed incapacitated to provide informed consent. Non-voluntarily consenting clients were excluded from the study.

\section{Data Sources/Measurement}

Primary data was obtained from participants using a structured interviewer-administered survey questionnaire. Information was gathered on the communication mediums available for clients' and health professionals' interactions and possible challenges emanating from the media used in Komfo Anokye Teaching Hospital (KATH). Three different questionnaires were designed and used. Each was used independently on the three different populations (health workers, clients and directorate managers). The reason was that each category has different ways of interacting within the hospital hence the different measuring tools. Prior to the data collection, the questionnaire was pre-tested at the Kwame Nkrumah University of Science and Technology (KNUST) hospital located in Kumasi to ensure the validity and reliability of the data to be collected from the study. Responses, opinions, and views generated from the pre-testing exercise were used as a guideline to review and refine the data collection tools used in the study.

\section{Recruitment of subjects}

Health professionals were randomly contacted during working hours of the day. The study processes (rationale, risks and benefits) were explained to them. Managers of the hospital who met the inclusion criteria were pre-informed of the study. This was followed by a scheduled meeting to explain the study and their respective consents were sought. Clients were randomly sampled during their out-client visits. Detailed information about the study was provided to them and their consents sought. Participants who consented voluntarily were recruited in the study.

\section{Sample size estimation}

Sample sizes were estimated for two different populations (health professionals and clients) using the following formula, .Proportions ( $p$ ) of $50 \%$ of clients' population and $50 \%$ of health professionals' population with experience of communication at the hospital were assumed respectively. With a $95 \%$ 
confidence interval ( $z=1.96$ for $95 \%), 6 \%$ margin of error, and $10 \%$ non-response rate, a sample size of 303 was estimated for each population (health professionals and clients). For the management members, 44 of them were purposively selected since it was not difficult knowing their exact number.

\section{Data collection and analysis}

Data collection in this study included variables for communication mediums used such as emails, letters, text messaging, phone calls, and direct interactions. Effectiveness of media as variable was described to respondents as the extent to which the communication media used are able to achieve the desired goals within the context of their operations (healthcare management, internal communication and clientphysician engagement). Open Data Kit (ODK) was used to collect the data. The data was cleaned and imported into STATA Version 16.0 (StataCorp. 4905 Lakeway Drive Station, Texas 77845, USA) for statistical analysis. Descriptive statistics were performed for all variables as proportions, and presented using tables.

\section{Results}

\section{Communication Mediums as Examined by Clients}

Table 1 present results of mediums used by clients for communications before coming (External), and within the hospital (internal).

Out of the 303 clients interviewed on the mediums for internal communications, a few $(n=8,2.64 \%)$ identified emails as the usual media for communicating with care providers, and majority ( $n=248,81.8 \%$ ) indicated they normally used direct means (Face-to-face) for all enquiries at the hospital. About $14.2 \%$ $(n=43)$ clients preferred communicating through meetings with health professionals whereas $10.6 \%$ $(n=32)$ usually communicated with professionals through social media (mainly WhatsApp). A proportion of about $4.3 \%(n=13)$ clients indicated that they normally communicate with providers through Letters/Notes in hand, especially when referred from other facilities, and $11.5 \%(n=35)$ of them usually used mobile phone calls for interacting with their care provider (Doctor) within the hospital.

With respect to the type mediums used for external (before hospital visits) communications, few clients $(n=8,2.6 \%)$ opted for emails as the usual means of communicating with providers. A section of about $28 \%(n=85)$ preferred having direct contact (face-to-face) with care provider before the scheduled visit date. About $5.3 \%(n=16)$ of clients normally used social media for discussions and enquiries, and $26.4 \%$ $(n=80)$ indicated that they usually used mobile phone calls to contact the hospital before coming to the hospital.

Assessment of the effectiveness of the mediums employed during external communication shows $29.4 \%$ $(n=89)$ of respondents viewing the mediums to be effective (Table 1$)$.

\section{Communication Mediums as Examined by Health Workers}


The mediums used for staff-to-management communications, staff-to-staff communications and staffto-client communications were examined and reported in table 2 . It also shows a detailed description and rating of the performance of the various mediums used for communication.

Among the various mediums assessed, memos was observed to be used by more than half $(n=171$, $56.4 \%)$ of the staff while $5.9 \%(n=8)$ used emails to interact with management of the hospital. Close to ninety percent $(n=, 272,89.8 \%)$ of the staff resorted to face-to-face as a medium to communicate with each other and about $97.4 \%(n=295)$ communicate with their clients by face-to-face (Table 2).

\section{Communication Mediums as Examined by Management}

The views of management members was sought on the purpose and various mediums or channels used for communication at the hospital and reported in table 3 . Half $(n=22,50 \%)$ of the management agreed that the current mediums were used to notify staff of changes in the hospital. Close to half $(n=21,47.7 \%)$ of the management agreed that the current mediums were used to notify the public and clients on the available services at the hospital, while $38.6 \%(n=17)$ disagreed to that. About $45.5 \%(n=20)$ of the management agreed that messages sent through the mediums were relevant whiles $38.6 \%$ were in disagreement to that. More than sixty percent $(n=28,63.6 \%)$ and almost half $(n=21,47.7)$ of the management agreed that messages sent through the mediums were consistent and credible respectively.

Respondents indicated some mechanisms used for disseminating feedbacks at the hospital as follows; feedback through surveys ( $n=16,36.4 \%)$, feedback through anonymous response cards $(n=15,34.1 \%)$, feedbacks through direct contact with supervisors $(n=28,63.6 \%)$ and feedbacks through suggestion boxes $(n=17,38.64 \%)$. About one third of the management $(n=15,34.1 \%)$ rated the communication mediums as effective in gathering feedbacks.

Respondents shared their views on the effectiveness of the various communication mediums in table 4 as follows; Emails were identified as effective sometimes ( $n=24,54.5 \%)$ while face-to-face medium which was noted by $45 . \%(n=20)$ respondents as effective all the time. Meetings and social media were noted as effective sometimes by $68.2 \%(n=30)$ and $61.4 \%(n=27)$ of management members respectively, while $22.7 \%(n=10)$ endorsed newsletters as effective means of communication (table 4).

With regards to efficiency of the mediums used, $56.8 \%(n=25)$ and $45.5 \%(n=20)$ of the respondents viewed email and face-to-face respectively as efficient sometimes. Again, meetings and social media were noted by $59.1 \%(n-26)$ and $56.8 \%(n=25)$ respectively as efficient and $52.3 \%(n=23)$ cited newsletter as efficient sometimes. Telephone and memos usage were indicated as efficient sometimes by $65.9 \%$ $(n=29)$ and $61.4 \%(n=27)$ respondents respectively. Generally, the communication mediums used at the hospital was rated by $38.6 \%(n=17)$ of the management as good (Table 5).

\section{Discussion}


This study examined the communication mediums used by health workers, management and clients who access care from the Komfo Anokye Teaching Hospital in Kumasi, Ghana.

This study stems from the possibility that communication media may vary the meaning and the sense of the information that is been transferred if not used in the right perspective(11). That is, in some settings across the globe, mobile phone communication has proven to be effective in information sharing among healthcare professionals $(3,4)$ in recent years. However, this result is not consistent across the entire spectrum of healthcare providers in different settings. In other jurisdictions, there is greater reliance on face-to-face approach as a medium of communication in hospitals though the growing digitization and technological advances threatens the continuing reliance on face-to-face interaction for medical and healthcare provision at all times(12). The high figures reported on face-to-face in this current study in Kumasi, Ghana during client-staff (81.9\%) and staff-staff (89.8) interactions as the most commonly identified medium of communication is in tandem with the global trend The adoption and preference for face-to-face in the health communication space is not unique to Ghana but has both a global reflection and more pronounced in many health sector establishments in many resource limited settings as observed by Travers in 2016 (12) .

Although face-to-face medium appears to be the ideal and rational means of communication in Ghana, it does not adequately address the communication problems in the health system (12) especially among clients with speech and hearing impairment (7) (13). The unevenness in the use of face-to-face communication as the preferred communication medium among healthcare providers has been documented in previous studies by Niemi and colleagues. Health workers and clients in those countries make use of ICT for communications using mediums such as the emails and text messages in their routine work (13). This gives clients such as those with speech and hearing impairment the chances to communicate better during seeking of health care. This observation runs contrary to the finding made in our present study. The study found very low preference for the use of electronic communication medium such as emails during interactions among clients-staff (2.6\%), staff-staff $(11.6 \%)$ and staff-management (5.9\%) respectively despite the acknowledged importance over other mediums of communication . Reasons for the non-frequent use of electronic communication media for healthcare seeking and management were not explored despite the acknowledgement of the role low level of ICT skills play in adopting electronic communication for healthcare among clients, hospital managers and hospital staff. Nonetheless as reported in previous studies, communicating using electronic means such as emails ahead of others gives the ability to revert to pending tasks (13). Likewise, one can take care of matters by email when it's convenient for you and as a matter of convenience (13)

The real burden of using inefficient medium of communication and its challenges to clients and health professionals was not a major priority under the scope of this study and should be investigated. However, the revelations from this study reinforce the need to incorporate electronic communication into the current health system in Ghana particularly in Teaching Hospitals and other referral hospitals.

\section{Conclusion}


The mediums clients used for enquiries from the hospital were mainly telephones. Within the hospital, clients' communication was however limited to verbal via face-to-face. Staffs mostly used social media and face-to-face method to interact with their colleague staffs. However, they communicated with clients by face-to-face only. Management also used memos more often to communicate with staffs and clients than the traditional verbal means via face-to-face. The current mediums of communication allowed management to notify staffs of relevant administrative changes and other information in the hospital. However, getting feedback through the same mediums seemed poor.

There exist heterogenous communication mediums used during internal and external communication. Heterogenous system of communication may lead to lack of information and or truncation of information flow. Developing a systematic way of information using a common information platform will improve access to health.

\section{Limitation of the study}

The descriptive exploratory nature of the study did not allow for the testing of associations and the conduct of robust quantitative analysis. Another limitation of the study stems from the possibility of social desirability bias. This is because, participants may under-report or overreport because they may either feel shy or fear to openly talk to data collection team. The study was done at only one tertiary hospital. Hence, extrapolating the findings to the entire healthcare system will not be entirely correct. Also, the individual respondents may have their own opinion on communication and may affect their responses.

\section{Abbreviations}

CHRPE -Committee of Human Research, Publications and Ethics

KReF $\quad$ KNUST Research Fund

KNUST $\quad$ Kwame Nkrumah University of Science and Technology

KATH -Komfo Anokye Teching Hospital

ICT - Information and Communication Technology

ODK -Open Data Kit

\section{Declarations}

\section{Ethical Approval and Consent to Participate}

Written approvals were respectively obtained from the Komfo Anokye Teaching Hospital and the Committee on Human Research, Publications and Ethics (CHRPE) of KNUST with registration number 
CHRPE/AP/591/18 before the study was conducted.

Participants read, understood and signed a written informed consent to be part of the study. The consent form contained detailed information of the study and participants were given enough time to make an informed decision before enrolling on the study.

\section{Consent for Publication}

Participants also consented to the publication of this work when the need arises.

\section{Availability of data and material}

The datasets generated during the current study are available from the corresponding author on reasonable request.

\section{Competing interest}

The authors declare that they have no competing interests.

\section{Funding}

Funding for the study was provided by KNUST Research Fund (KReF).

\section{Authors' contribution}

DA, MD, FAO and SN designed the study, JB, EKA, KL, EXA, AA, DA, BAD, AKO, SCYAP and NKM collected the data, cleaned and analyzed the data. MD and FAO drafted the initial manuscript and was reviewed by all authors. All authors read and approved the final manuscript and are accountable for the content.

\section{Acknowledgement}

We would like to thank all the health workers and clients who took out time from their schedule to participate in our study.

\section{References}

1. WHO, OECD TWB. Delivering quality health services. 2018. 100 p.

2. Gog. the Ghana Ict for Accelerated Development. Econ Soc. 2003;(June):1-85.

3. Kim MS, Aro MR, Lage KJ, Ingalls KL, Sindhwani V, Markey MK. Exploring the Usability of Mobile Apps Supporting Radiologists' Training in Diagnostic Decision Making. J Am Coll Radiol. 2016;13(3):335-43.

4. Ventola CL. Mobile devices and apps for health care professionals: uses and benefits. PT. 2014;39(5):356-64. 
5. BOATENG MR. MS. the Role of Information and Communication Technologies in Ghana'S Rural Development. 2012;

6. Hanchate AD, Paasche-Orlow MK, Baker WE, Lin M-Y, Banerjee S, Feldman J. Association of Race/Ethnicity With Emergency Department Destination of Emergency Medical Services Transport. JAMA Netw Open. 2019 Sep;2(9):e1910816.

7. Abdur Rahman Alfa Shaban. Lack of beds forces Ghana's main hospital to reject critical referrals | Africanews [Internet]. 2018 [cited 2018 Oct 11]. Available from: http://www.africanews.com/2018/07/11/lack-of-beds-forces-ghanas-main-hospital-to-reject-criticalreferrals/

8. Raifman JRG, Lanthorn HE, Rokicki S, Fink G. The impact of text message reminders on adherence to antimalarial treatment in northern ghana: A randomized trial. PLoS One. 2014 Oct;9(10).

9. Abor PA. Exploring clinical communication in a teaching hospital in Ghana. Int J Heal Gov. 2019;24(2):155-68.

10. Population and housing census. Kumasi metropolitan. 2010;92.

11. MMEDIUM THEORY. Communication theory.org. 2020.

12. Travers C. DOES THE FUTURE HAVE ROOM FOR FACE TO FACE COMMUNICATION. 2016.

13. Niemi A, Hupli M, Koivunen M. The use of electronic communication for client-professional interaction - nursing staff 's point of view. FinJeHeW fininish J eHealth eWelfare. 2016;8(4).

\section{Tables}

Table 1: communication Mediums as Examine by Patients 


\begin{tabular}{|c|c|c|c|}
\hline \multicolumn{2}{|c|}{ VARIABLE } & $\begin{array}{l}\text { No } \\
(n=303)\end{array}$ & $\begin{array}{l}\text { Yes } \\
(n=303)\end{array}$ \\
\hline \multicolumn{4}{|c|}{ Mediums used for communication at the hospital. } \\
\hline- & Email & 97.4 & 2.6 \\
\hline- & Face-to-Face & 18.1 & 81.9 \\
\hline- & Meetings & 85.8 & 14.2 \\
\hline- & Social media & 89.4 & 10.6 \\
\hline- & Newsletters/Letters & 95.7 & 4.3) \\
\hline- & Telephone & 88.5 & 11.5 \\
\hline \multicolumn{4}{|c|}{ Mediums used to contact the hospital before coming } \\
\hline- & Email & 97.4 & 2.6 \\
\hline - & Face-to-Face & 72 & 28 \\
\hline- & Meetings & 95.7 & 4.3 \\
\hline- & Social media & 94.7 & 5.3 \\
\hline- & Newsletters/Letters & 94.4 & 5.6 \\
\hline- & Telephone & 73.6 & 26.4 \\
\hline- & No medium used & 56.8 & 43.2 \\
\hline \multicolumn{4}{|c|}{ Effectiveness of communication medium used before coming to the hospital } \\
\hline- & very effective & 18 & 5.9 \\
\hline- & effective & 89 & 29.4 \\
\hline- & a little effective & 45 & 14.9 \\
\hline- & not at all effective & 20 & 6.6 \\
\hline- & very effective & 34 & 11.2 \\
\hline - & not at all effective & 31 & 10.2 \\
\hline
\end{tabular}

Table 2 :Communication Mediums as Examined by Health Workers 


\begin{tabular}{lcc}
\hline VARIABLES & $\begin{array}{c}\text { YES } \\
\text { (\%) }\end{array}$ & $\begin{array}{c}\text { NO } \\
(\%)\end{array}$ \\
\hline Staff-to-management & & \\
Meetings & 64.4 & 35.6 \\
\hline Memo & 56.4 & 43.6 \\
\hline Letter & 51.5 & 48.5 \\
\hline Face-to-Face & 50.2 & 49.8 \\
\hline Telephone & 42.2 & 57.8 \\
\hline Social-Media & 16.2 & 83.8 \\
\hline Email & 5.9 & 94.1 \\
\hline Staff-to-staff & & \\
\hline Face-to-Face & 89.8 & 10.2 \\
\hline Telephone & 66.0 & 34.0 \\
\hline Meetings & 65.0 & 35.0 \\
\hline Social-Media & 54.8 & 45.2 \\
\hline Memo & 38.9 & 61.1 \\
\hline Newsletter/letter & 28.7 & 71.3 \\
\hline Email & 11.5 & 88.5 \\
\hline Staff-to-patient & & \\
\hline Face-to-Face & 97.4 & 2.6 \\
\hline Telephone & 42.6 & 57.4 \\
\hline Email & 2.0 & 98 \\
\hline Meetings & 20.5 & 79.5 \\
\hline Social-Media & 19.8 & 80.2 \\
\hline Newsletter/letter & 14.2 & 85.8 \\
\hline & & \\
\hline
\end{tabular}

Source: Field Data (2018)

Table 3: Communication Mediums as Examined by Management 


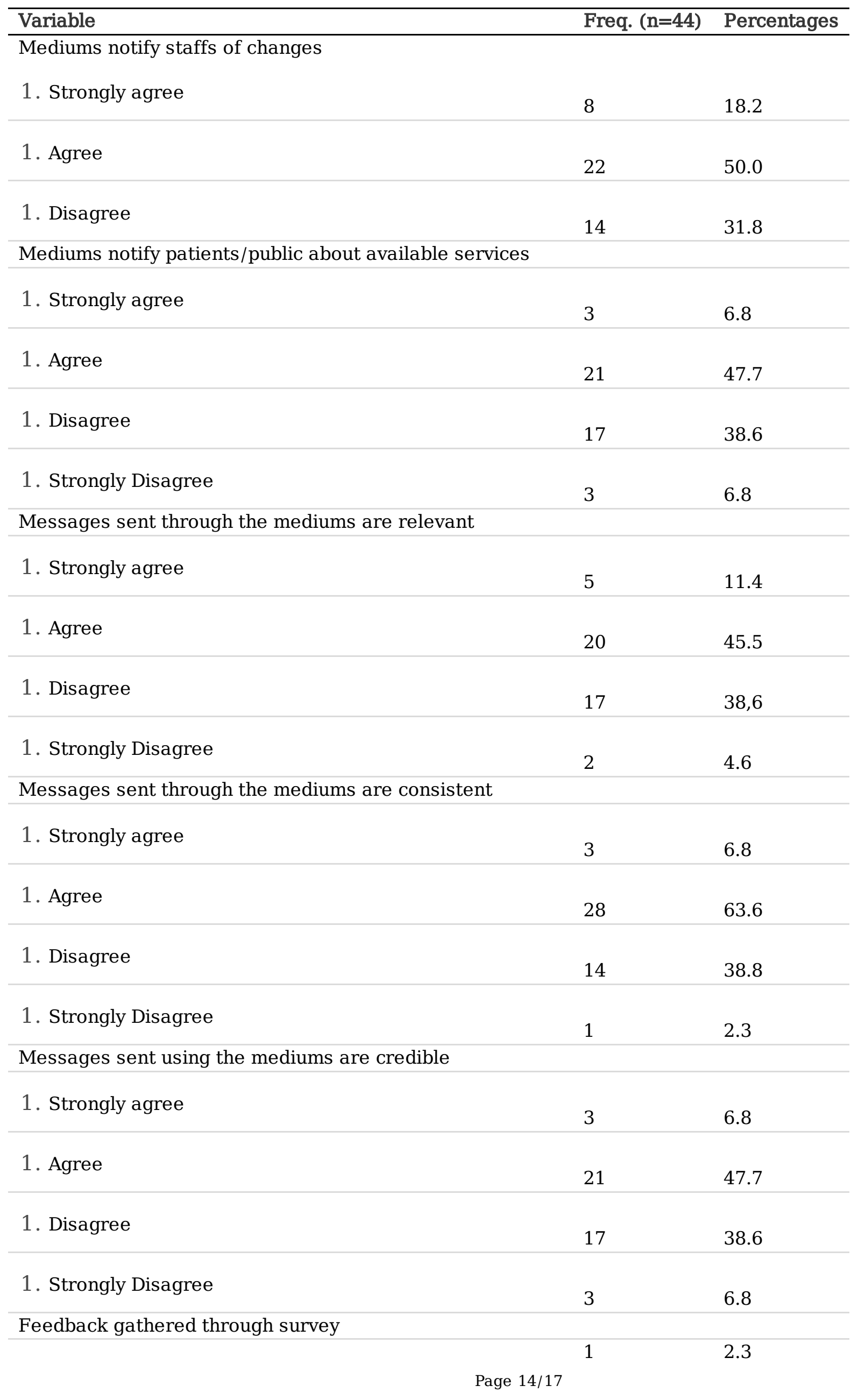


1. Stronglyagree

1. Agree

1. Disagree

1. Strongly Disagree

Feedback gathered through anonymous response cards

1. Strongly agree

1. Agree

1. Disagree

1. Strongly disagree

Feedback gathered through direct contact with supervisors

1. Strongly agree

1. Agree

Disagree

1. Strongly disagree
16

36.4

21

47.7

6

13.6

4.6

15

34.1

21

52.3

4

9.1

15.9

7

63.6

18.2

$\begin{array}{ll}28 & 63.6 \\ 8 & 18.2\end{array}$

1

2.3

Source: Field Data 2018

Table 4: Communication Mediums Effectiveness as Examined by Management 


\begin{tabular}{|c|c|c|c|}
\hline \multirow{2}{*}{\multicolumn{2}{|c|}{$\begin{array}{l}\text { Variable } \\
\text { Medium is effective for feedback }\end{array}$}} & \multirow[t]{2}{*}{ Freq. $(\mathrm{n}=44)$} & \multirow[t]{2}{*}{ Percentages } \\
\hline & & & \\
\hline- & Strongly agree & 3 & 6.8 \\
\hline - & Agree & 15 & 34.1 \\
\hline- & Disagree & 25 & 56.9 \\
\hline- & Strongly disagree & 1 & 2.3 \\
\hline \multicolumn{2}{|c|}{ Effectiveness of Email as a channel } & \multirow[t]{2}{*}{6} & \multirow[t]{2}{*}{13.6} \\
\hline- & All the time & & \\
\hline- & Sometimes & 24 & 54.5 \\
\hline- & Not at all & 14 & 31.8 \\
\hline \multicolumn{4}{|c|}{ Effectiveness of Face-to-Face as a channel } \\
\hline- & All the time & 20 & 45.4 \\
\hline- & Sometimes & 19 & 43.2 \\
\hline - & Not at all & 5 & 11.4 \\
\hline \multicolumn{4}{|c|}{ Effectiveness of Meeting as a channel } \\
\hline- & All thetime & 12 & 27.3 \\
\hline- & Sometimes & 30 & 68.2 \\
\hline- & Not at all & 2 & 4.55 \\
\hline \multicolumn{4}{|c|}{ Effectiveness of Social Media as a channel } \\
\hline- & All the time & 9 & 20.5 \\
\hline- & Sometimes & 27 & 61.4 \\
\hline- & No at all & 8 & 18.2 \\
\hline \multicolumn{4}{|c|}{ Effectiveness of Newsletters as a channel } \\
\hline- & All the time & 10 & 22.7 \\
\hline- & Sometimes & 18 & 40.9 \\
\hline- & Not at all & 16 & 45.4 \\
\hline \multicolumn{4}{|c|}{ Effectiveness of telephone as a channel } \\
\hline- & All the time & 11 & 25 \\
\hline- & sometimes & 27 & 61.4 \\
\hline- & Not at all & 6 & 13.6 \\
\hline
\end{tabular}

Table 5: Communication Mediums Effectiveness as Examined by Management 


\begin{tabular}{|c|c|c|c|}
\hline \multirow{2}{*}{\multicolumn{4}{|c|}{$\begin{array}{l}\text { Variable } \\
\text { Emails are Efficient }\end{array}$}} \\
\hline & & & \\
\hline- & All the time & 5 & 11.4 \\
\hline- & Sometimes & 25 & 56.8 \\
\hline- & Not at all & 14 & 31.8 \\
\hline \multicolumn{4}{|c|}{ Face to Face is Efficient } \\
\hline- & All the time & 15 & 34.1 \\
\hline- & Sometimes & 20 & 45.5 \\
\hline- & Not at all & 9 & 20.4 \\
\hline \multicolumn{4}{|c|}{ Meetings are Efficient } \\
\hline- & All the time & 10 & 22.7 \\
\hline- & Sometimes & 26 & 59.1 \\
\hline- & Not at all & 8 & 18.2 \\
\hline \multicolumn{4}{|c|}{ Social media is Efficient } \\
\hline- & All the time & 12 & 27.3 \\
\hline- & Sometimes & 25 & 56.8 \\
\hline- & Not at all & 7 & 15.9 \\
\hline \multicolumn{4}{|c|}{ Newsletters are Efficient } \\
\hline- & All the time & 8 & 18.2 \\
\hline- & Sometimes & 23 & 52.3 \\
\hline- & Not at all & 13 & 29.5 \\
\hline \multicolumn{4}{|c|}{ Telephone use is Efficient } \\
\hline- & All the time & 10 & 22.7 \\
\hline- & Sometimes & 29 & 65.9 \\
\hline- & Not at all & 5 & 11.2 \\
\hline \multicolumn{4}{|c|}{ Memo is Efficient } \\
\hline- & All the time & 14 & 31.8 \\
\hline- & Sometimes & 327 & 61.4 \\
\hline- & Not at all & 3 & 6.8 \\
\hline \multicolumn{4}{|c|}{ General performance of mediums } \\
\hline- & Excellent & 5 & 11.4 \\
\hline- & Good & 17 & 38.6 \\
\hline- & Poor & 7 & 15.9 \\
\hline- & Very good & 11 & 25 \\
\hline- & Very poor & 4 & 9.1 \\
\hline
\end{tabular}

Source: Field data; 2018

\section{Supplementary Files}

This is a list of supplementary files associated with this preprint. Click to download.

- Formula.docx 